Marshall University

Marshall Digital Scholar

Physics Faculty Research

Physics

8-1-2002

\title{
Competition between ferromagnetic metallic and paramagnetic insulating phases in manganites
}

G. Li

H. D.Zhou

S.J. Feng

Xiaojuan Fan

Marshall University, fan2@marshall.edu

X. G. Li

See next page for additional authors

Follow this and additional works at: http://mds.marshall.edu/physics_faculty

Part of the Condensed Matter Physics Commons

\section{Recommended Citation}

Li, G., Zhou, H. D., Feng, S. J., Fan, X. J., Li, X. G., \& Wang, Z. D. (2002). Competition between ferromagnetic metallic and paramagnetic insulating phases in manganites. Journal of Applied Physics, 92(3), 1406-1410.

This Article is brought to you for free and open access by the Physics at Marshall Digital Scholar. It has been accepted for inclusion in Physics Faculty Research by an authorized administrator of Marshall Digital Scholar. For more information, please contact zhangj@marshall.edu. 
Authors

G. Li, H. D. Zhou, S. J. Feng, Xiaojuan Fan, X. G. Li, and Z. D. Wang 


\title{
Competition between ferromagnetic metallic and paramagnetic insulating phases in manganites
}

\author{
G. Li, H.-D. Zhou, S. J. Feng, X.-J. Fan, and X.-G. Li ${ }^{\text {a) }}$ \\ Structure Research Laboratory, Department of Materials Science and Engineering, University of Science \\ and Technology of China, Hefei 230026, People's Republic of China \\ Z. D. Wang \\ Department of Physics, University of Hong Kong, Pokfulam Road, Hong Kong, and \\ Structure Research Laboratory, Department of Materials Science and Engineering, University of Science \\ and Technology of China, Hefei 230026, People's Republic of China
}

(Received 29 October 2001; accepted for publication 8 May 2002)

\begin{abstract}
$\mathrm{La}_{0.67} \mathrm{Ca}_{0.33} \mathrm{Mn}_{1-x} \mathrm{Cu}_{x} \mathrm{O}_{3}$ ( $x=0$ and 0.15 ) epitaxial thin films were grown on the (100) $\mathrm{LaAlO}_{3}$ substrates, and the temperature dependence of their resistivity was measured in magnetic fields up to $12 \mathrm{~T}$ by a four-probe technique. We found that the competition between the ferromagnetic metallic (FM) and paramagnetic insulating (PI) phases plays an important role in the observed colossal magnetoresistance (CMR) effect. Based on a scenario that the doped manganites approximately consist of phase-separated FM and PI regions, a simple phenomenological model was proposed to describe the CMR effect. Using this model, we calculated the resistivity as functions of temperature and magnetic field. The model not only qualitatively accounts for some main features related to the CMR effect, but also quantitatively agrees with the experimental observations. (C) 2002 American Institute of Physics. [DOI: 10.1063/1.1490153]
\end{abstract}

\section{INTRODUCTION}

The perovskite manganites $\mathrm{La}_{1-x}(\mathrm{Ca}, \mathrm{Sr}, \mathrm{Ba})_{x} \mathrm{MnO}_{3}$, in the doping region $x \sim 0.2-0.4$, exhibit a transition from a paramagnetic insulator (PI) to a ferromagnetic metal (FM) on cooling. This transition can be modified by an external magnetic field producing negative "colossal magnetoresistance" (CMR). ${ }^{1}$ Ferromagnetism and the PI-FM transition in these materials are usually attributed to the double exchange mechanism. ${ }^{2}$ However, recent calculation shows that the double exchange mechanism alone cannot explain the CMR effect. ${ }^{3}$ More and more studies have indicated that a microscopic phase separation plays an essential role in the physics of the manganites. ${ }^{4-7}$ In particular, it results in the apparent percolative character of the insulator-metal transition when the transition is from the charge-ordered insulating to the ferromagnetic metallic state. ${ }^{8}$

Although much work was devoted to the physical properties of manganites, the microscopic nature of the phaseseparated states has been far from well understood. For example, how the electronic properties of the constituent phases as well as their volume fractions and spatial distributions are dependent on the temperature and external magnetic fields remains to be characterized.

There was a two-component phenomenological model, describing polaron formation in CMR compounds, devised by Jaime et al. ${ }^{9}$ and used by Rubinstein to quantitatively analyze the transport properties and Hall coefficient of $\mathrm{La}_{2 / 3} \mathrm{Ca}_{1 / 3} \mathrm{MnO}_{3} .{ }^{10}$ Recently, Yuan et al. ${ }^{11}$ supposed that the conducting path of the CMR materials consists of a series of

\footnotetext{
a) Author to whom correspondence should be addressed; electronic mail: lixg@ustc.edu.cn
}

paramagnetic and ferromagnetic regions but the physical image is yet to be clarified.

In this article, we focused on the study of resistivity as functions of temperature and magnetic field, and intended to elucidate the role of competition between the FM and PI phases. It was approximated that the two main separated phases coexist in the doped perovskite manganese oxides: one is the paramagnetic insulating phase, and the other is the ferromagnetic metallic phase, with the energy difference to form the two phases. Under this approximation, the temperature dependence of the volume fraction distribution is supposed to follow the Boltzmann distribution of a two energylevel system then we presented a simple model to describe the PI-FM transition and CMR. In order to test the feasibility of our model, we fabricated high quality $\mathrm{La}_{0.67} \mathrm{Ca}_{0.33} \mathrm{Mn}_{1-x} \mathrm{Cu}_{x} \mathrm{O}_{3}(x=0$ and 0.15 ) epitaxial thin films which exhibit CMR effect. Detailed analysis shows that the model is quantitatively consistent with our experimental observations.

\section{EXPERIMENT}

The sputtering targets, with a nominal composition of $\mathrm{La}_{0.67} \mathrm{Ca}_{0.33} \mathrm{Mn}_{1-x} \mathrm{Cu}_{x} \mathrm{O}_{3}$ ( $x=0$ and 0.15 ), were prepared using a sol-gel technique. X-ray diffraction (XRD) patterns recorded by MacScience MAXP18AHF diffractometer using $\mathrm{Cu} K \alpha$ radiation show that they have a crystal structure close to cubic perovskite with a lattice constant $a \approx 0.388 \mathrm{~nm}$, as shown in Fig. 1(a). We grew epitaxial $\mathrm{La}_{0.67} \mathrm{Ca}_{0.33} \mathrm{Mn}_{1-x} \mathrm{Cu}_{x} \mathrm{O}_{3}$ [x=0 (LCMO) and 0.15 (LC$\mathrm{MCO})]$ thin films on the (100) $\mathrm{LaAlO}_{3}$ single-crystal substrate with a magnetron sputtering technique. The thickness of the annealed films is about $300 \mathrm{~nm}$. X-ray diffraction 

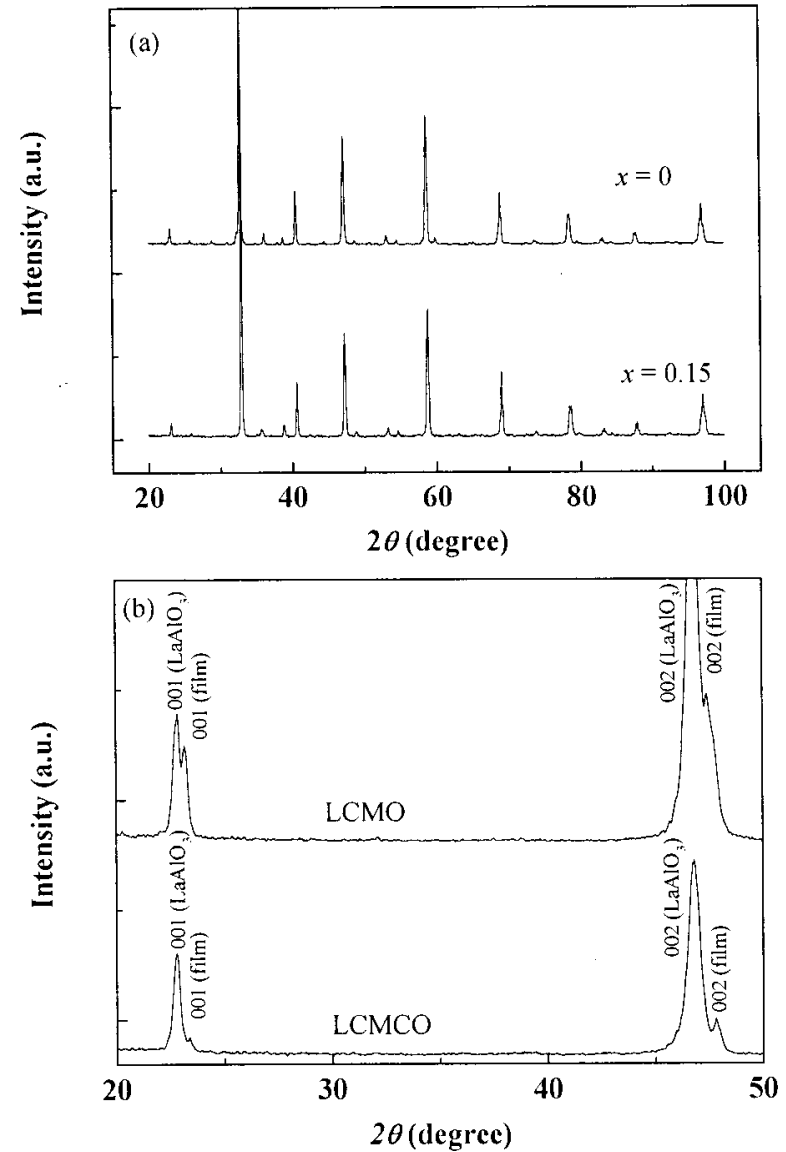

FIG. 1. X-ray patterns for $\mathrm{La}_{0.67} \mathrm{Ca}_{0.33} \mathrm{Mn}_{1-x} \mathrm{Cu}_{x} \mathrm{O}_{3}(x=0$ and 0.15$)$ system: (a) polycrystalline and (b) epitaxial films mounted on (100) $\mathrm{LaAlO}_{3}$ substrates.

analysis shows that the films have a single phase orientated along $c$ axis with lattice parameter $c \approx 0.391$ and $0.3885 \mathrm{~nm}$ for LCMO and LCMCO, respectively, as depicted in Fig. $1(b)$.

Figure 2(a) shows the ac susceptibility for the polycrystalline samples. It can be seen that the paramagnetic to ferromagnetic transition temperatures $\left(T_{c}\right)$ for $x=0$ and 0.15 samples are 268 and $234 \mathrm{~K}$, respectively. The dc magnetization measured by a BHV-55 Vibrating Sample Magnetometer (VSM, Richen Denshi) with 1000 Oe applied magnetic field parallel to the $a b$ plane of LCMCO film reveals that it also has a $T_{c}$ equal to that of the $x=0.15$ target, as can be seen in Fig. 2(b).

The temperature dependencies of resistivity for the films were measured in magnetic fields up to $12 \mathrm{~T}$ upon warming from $4.2 \mathrm{~K}$ to room temperature by a four-probe technique, as the symbols shown in Fig. 3. It can be seen that the films experience a semiconductor-metal transition at $T_{P}(\approx 247$ and 215.7 K for LCMO and LCMCO, respectively), above or below which a semiconductor-like transport or a metallic behavior is clearly seen in the absence of magnetic field. Applied magnetic fields drive the semiconductor-metal transition to high temperatures and the CMR effect appears. Note that because $T_{P}$ of LCMO moves to high temperature range $(>300 \mathrm{~K})$ when the magnetic fields exceed $4 \mathrm{~T}$, we only present $\rho$ vs $T$ curves in magnetic fields of 0 and $4 \mathrm{~T}$ for the

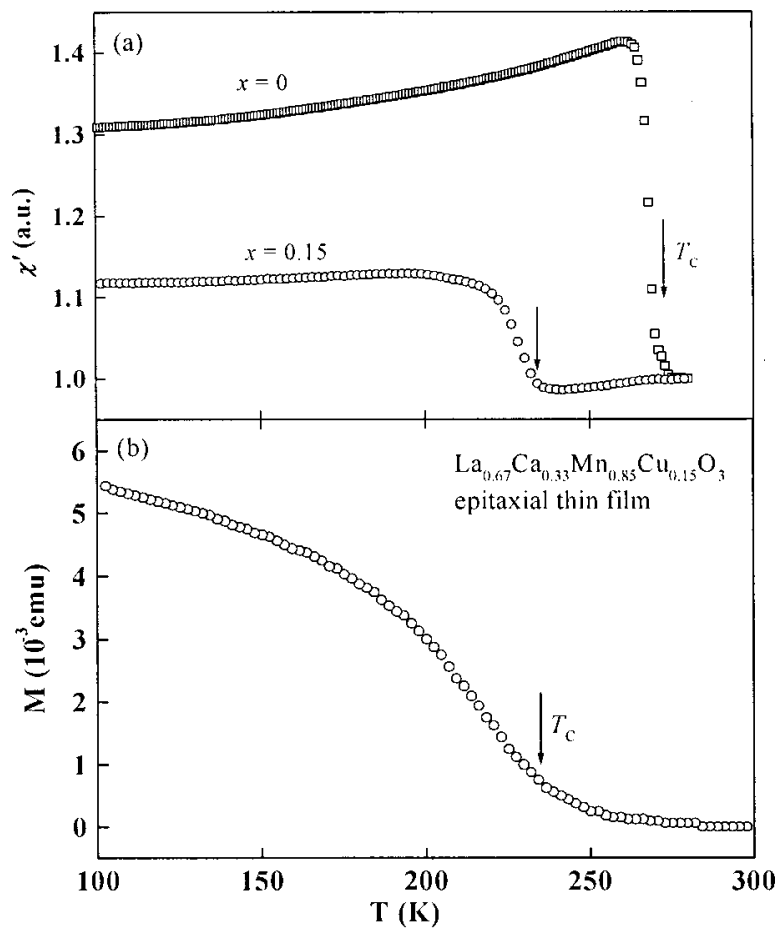

FIG. 2. ac and dc magnetization curves as a function of temperature for (a) $\mathrm{La}_{0.67} \mathrm{Ca}_{0.33} \mathrm{Mn}_{1-x} \mathrm{Cu}_{x} \mathrm{O}_{3} \quad(x=0$ and 0.15$)$ polycrystalline, and (b) $\mathrm{La}_{0.67} \mathrm{Ca}_{0.33} \mathrm{Mn}_{0.85} \mathrm{Cu}_{0.15} \mathrm{O}_{3}$ epitaxial thin film. The arrows point to the Curie temperature.

film. The residual resistivity $\left(\rho_{0}\right)$ both for LCMO and LCMCO changes slightly even when the magnetic field is 4 and $12 \mathrm{~T}$, as listed in Table I. XRD, dc magnetization, and transport properties in magnetic fields demonstrate that the films are of high quality.

\section{MODEL AND DISCUSSION}

Magnetization measurements have shown that the manganese oxides having CMR effect often experience a paramagnetic to ferromagnetic transition accompanied by an insulator-metal transition during the cooling procedure. Electron resonance experiments have further confirmed that just above Curie temperature ${ }^{12}$ the system shows a heterogeneous magnetism (paramagnetic and ferromagnetic phases coexist), and it will reach a completely homogeneous paramagnetic phase when temperature is much higher than Curie temperature $T_{c}$. Uehara et al. ${ }^{13}$ reported that there is a phase separation including paramagnetic and ferromagnetic or insulator and metallic phases even when the system is cooled to temperature lower than its Curie temperature. Goodenough $^{14}$ indicated that in CMR materials, a metallic conductivity exists in the ferromagnetic regions (due to the double exchange mechanism) and a semiconductor-like conductivity in the paramagnetic regions at temperature higher than Curie temperature. These features enable us to suppose the materials to be composed of paramagnetic and ferromagnetic regions, and semiconductor-like transport properties are exhibited in the paramagnetic regions while metallic transports always show up in ferromagnetic regions. Neverthe- 

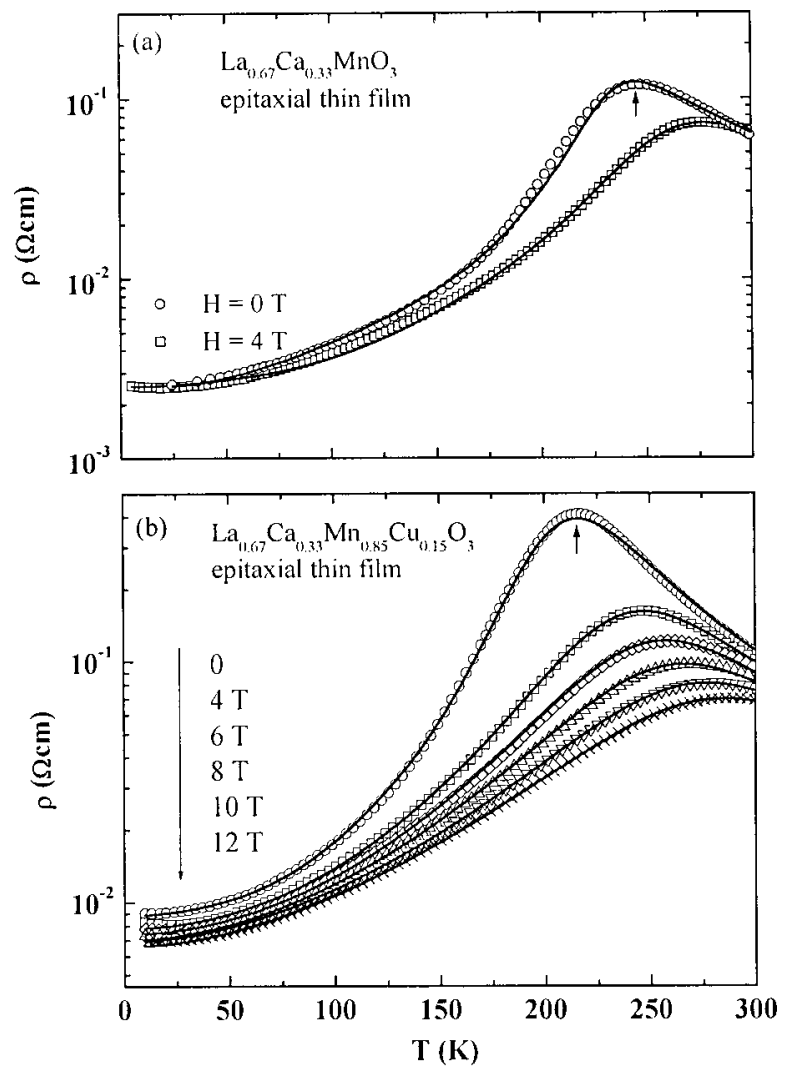

FIG. 3. Comparison between the calculated (solid lines) and experimental (symbols) temperature dependencies of resistivity of (a) $\mathrm{La}_{0.67} \mathrm{Ca}_{0.33} \mathrm{MnO}_{3}$, and (b) $\mathrm{La}_{0.67} \mathrm{Ca}_{0.33} \mathrm{Mn}_{0.85} \mathrm{Cu}_{0.15} \mathrm{O}_{3}$ epitaxial thin films in different magnetic fields up to $12 \mathrm{~T}$. The arrows denote $T_{P}$ positions.

less, how the volume fractions of the PI and FM regions as well as the total resistivity change with the temperature and magnetic field needs to be clarified.

Suppose $f$ and $f^{\prime}$ represent the volume fractions of PI and FM regions in the system, respectively, one apparently has the relation $f+f^{\prime}=1$. Physically, it is acceptable and reasonable to assume that there is an energy difference (per unit cell) $\Delta U$ between the FM state and PI state. As a result, the volume fractions obey a simple two energy-level Boltzmann distribution:

$$
f=\frac{1}{1+\exp \left(\Delta U / k_{B} T\right)},
$$

and

$$
f^{\prime}=1-f=\frac{\exp \left(\Delta U / k_{B} T\right)}{1+\exp \left(\Delta U / k_{B} T\right)} .
$$

Moreover, the total resistance of the system can be considered to be the sum of the resistances of the phase-separated FM and PI parts, and thus the whole resistivity $\rho$ of the system can be written as

$$
\rho=\rho_{\mathrm{FM}} \cdot f+\rho_{\mathrm{PL}} \cdot(1-f),
$$

where $\rho_{\mathrm{FM}}$ and $\rho_{\mathrm{PI}}$ are the resistivities for the FM and PI regions, respectively.

At $T=0 \mathrm{~K}$, the FM state is the ground state of the system and the PI state energy is higher, and the energy difference $(-\Delta U)$ reaches to the maximum $U_{0}$. As the FM state is a spin-ordered state, it is sensitive to the temperature. When temperature increases, some PI regions will appear, but the long-range FM order will be kept to a characteristic temperature $T_{C}^{\text {mod }}$ (here $T_{C}^{\text {mod }}$ is a PI-FM transition temperature used in the model and near/equal to $T_{c}$ ). Without loss of generality, we may expand $\Delta U(T)$ around $T_{C}^{\text {mod }}$ to the first order of $\left(T-T_{C}^{\mathrm{mod}}\right)$ and require that $\Delta U \approx 0$ at $T=T_{C}^{\mathrm{mod}}$. Therefore we may write

$$
\Delta U \approx-U_{0}\left(1-T / T_{C}^{\mathrm{mod}}\right),
$$

where $U_{0}$ can be considered as the energy difference for temperature well below $T_{C}^{\text {mod }}$ if we extrapolate Eq. (3) to low temperatures. From Eqs. (1) and (3), one can find that, (i) $f$ $\sim 1$ for $T<T_{C}^{\mathrm{mod}}$; (ii) $f^{\prime} \sim 1$ for $T>T_{C}^{\mathrm{mod}}$. Near $T_{C}^{\mathrm{mod}}$, due to the competition between the contributions of FM and PI regions, the resistivity tends to reach its maximum. ${ }^{15}$

As known, in the metallic conducting temperature region well below $T_{P}$, the metallic resistivity can be ascribed to the residual resistivity $\rho_{0}$, single-magnon's scattering contribution $A T^{216}$ and electron-phonon interaction $B T^{5}$, respectively, namely, $\rho_{\mathrm{FM}}=\rho_{0}+A T^{2}+B T^{5}$. The $T^{5}$ term is the correction from an electron-phonon scattering, which is also

\begin{tabular}{|c|c|c|c|c|c|c|c|c|}
\hline$H(\mathrm{~T})$ & $\begin{array}{c}\rho_{0} \\
(\mathrm{~m} \Omega \mathrm{cm})\end{array}$ & $\begin{array}{c}A \\
\left(\Omega \times \mathrm{cm} \mathrm{K}^{-2}\right)\end{array}$ & $\begin{array}{c}B \\
\left(\Omega \times \mathrm{cm} \mathrm{K}^{-5}\right)\end{array}$ & $C\left(\Omega \times \mathrm{cm} \mathrm{K}^{-1}\right)$ & $E_{g} / k_{B}(\mathrm{~K})$ & $T_{C}^{\bmod }(\mathrm{K})$ & $T_{P}(\mathrm{~K})$ & $U_{O} / k_{B}(\mathrm{~K})$ \\
\hline \multicolumn{9}{|c|}{ LCMO film } \\
\hline 0 & 2.5 & $1.52 \times 10^{-7}$ & $2.91 \times 10^{-14}$ & $1.54 \times 10^{-7}$ & 1704.2 & 241 & 245.2 & 4302 \\
\hline 4 & 2.5 & $0.83 \times 10^{-7}$ & $2.81 \times 10^{-14}$ & $1.54 \times 10^{-7}$ & 1465.2 & 270 & 276.8 & 4200 \\
\hline \multicolumn{9}{|c|}{ LCMCO film } \\
\hline 0 & 8.85 & $5.08 \times 10^{-7}$ & $4.0 \times 10^{-13}$ & $2.46 \times 10^{-6}$ & 1498.3 & 213.3 & 215.7 & 3420 \\
\hline 4 & 7.78 & $4.65 \times 10^{-7}$ & $1.50 \times 10^{-13}$ & $2.46 \times 10^{-6}$ & 1370.1 & 249.9 & 246.9 & 3381 \\
\hline 6 & 7.37 & $4.42 \times 10^{-7}$ & $1.03 \times 10^{-13}$ & $2.46 \times 10^{-6}$ & 1260.7 & 263.4 & 259.2 & 3353 \\
\hline 8 & 7.00 & $4.27 \times 10^{-7}$ & $0.70 \times 10^{-13}$ & $2.46 \times 10^{-6}$ & 1153.7 & 275.2 & 269.1 & 3305 \\
\hline 10 & 6.88 & $3.99 \times 10^{-7}$ & $0.48 \times 10^{-13}$ & $2.46 \times 10^{-6}$ & 1016.1 & 288.8 & 279.9 & 3260 \\
\hline 12 & 6.57 & $3.75 \times 10^{-7}$ & $0.36 \times 10^{-13}$ & $2.46 \times 10^{-6}$ & 698.8 & 300.7 & 289.4 & 3180 \\
\hline
\end{tabular}
employed to explain the low-temperature resistivity in $\mathrm{La}_{0.6} \mathrm{Ca}_{0.4} \mathrm{MnO}_{3}$ thin film. ${ }^{17}$ While in semiconductor-like

TABLE I. Parameters used to fit the experimental data of LCMO and LCMCO films. 
conducting temperature region well above $T_{P}$, the resistivity can be almost described by a formula as $\rho_{\mathrm{PI}}$ $=C T \exp \left(E_{g} / k_{B} T\right)$ in terms of a magnetic polaron picture. ${ }^{18}$ When a magnetic field is applied, the resistivity decreases and CMR effect appears. This can be qualitatively understood from Eqs. (1), (2), and (3). The coefficients $A$ and $B$ of $T^{2}$ and $T^{5}$ terms and the activation energy $E_{g}$ become small due to the suppression of single-magnon scattering in FM regions and the formation of polarons in PI regions in the magnetic field, respectively.

The most important effect induced by the external magnetic field is that the PI-FM transition temperature moves to a higher temperature, which has been observed or predicted by experiments or theoretical calculation. ${ }^{19}$ It means that the external magnetic field increases the FM fraction; part of the PI fraction is melt and turns into FM state with the help of the external field, liberating its portion of carriers and leading to the large enhancement of conductivity. As a result, the CMR effect manifestly appears. This is reasonable since the metallic component triggered by ferromagnetism is also sensitive to the magnetic field, and the sizes of FM clusters grow as magnetic field is applied, especially near the PI-FM transition temperature. Because $T_{C}^{\text {mod }}$ is near the PI-FM transition temperature, it is naturally expected that $T_{C}^{\mathrm{mod}}$ will also move to a higher temperature in the magnetic fields. To be more specific, we present the calculated resistivity for 0 and $4 \mathrm{~T}$ magnetic fields by using Eqs. (1), (2), and (3) (the parameters are listed in the caption of Fig. 4). It should be noted that, according to the above discussion, we only allow $A, B, E_{g}$, and $T_{C}^{\text {mod }}$ to change with the magnetic field. The temperature dependence of ferromagnetic volume fraction $f$ in 0 and $4 \mathrm{~T}$ magnetic fields is shown in Fig. 4(a). It is clear that $f$ is close to 1 at temperatures well below $T_{C}^{\bmod }$, and it approaches zero when the temperature increases well above $T_{C}^{\text {mod }}$. The calculated resistivity is plotted in Fig. 4(b). Note that the two figures refer to the LCMCO epitaxial thin film.

In order to verify our model, we employ it to analyze the magnetic transport data of LCMO and LCMCO epitaxial film. Indeed, it was found that the resistivity of LCMO and LCMCO films at temperatures lower or higher than $T_{P}$ can be almost fitted to $\rho_{0}+A T^{2}+B T^{5}$ and $C T \exp \left(E_{g} / k_{B} T\right)$, with the parameters $\rho_{0}, A, B, C$, and $E_{g}$ listed in Table I, respectively. Therefore, in the fitting for the whole temperature range, the parameters $\rho_{0}, A, B, C$, and $E_{g}$ are fixed as indicated in Table I, while $T_{C}^{\text {mod }}$ and $U_{0}$ are adjustable parameters for each curve. The solid lines in Fig. 3 show the fitting results. It can be seen that the results calculated from Eqs. (1), (2), and (3) agree well with the experimental data. As a result, $T_{C}^{\mathrm{mod}}$ is very close to the $T_{P}$ (the semiconductor-metal transition point). In addition, $T_{C}^{\bmod }$ as expected increases while $E_{g}$ decreases as the external magnetic field increases. The magnetic field-dependent $T_{P}, T_{C}^{\mathrm{mod}}, E_{g}$, and $U_{0}$ are shown in Figs. 5(a) and 5(b), respectively. In addition, it should be noted that if one uses the measured $T_{P}$ to replace $T_{C}^{\mathrm{mod}}$ in Eq. (3) and fits the measured data with only one adjustable parameter $U_{0}$, then the fitting also appears to be quite satisfactory (not shown here).

One may notice that the parameters used here, except for $C$ and $T_{C}^{\mathrm{mod}}$, are becoming smaller as the applied magnetic
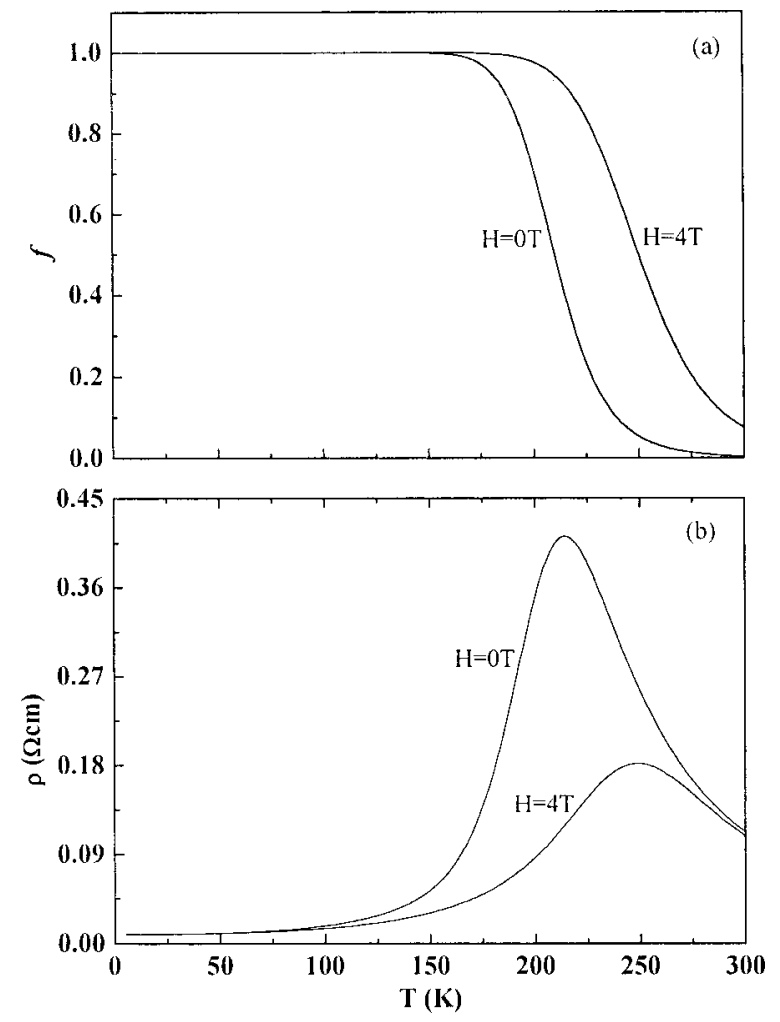

FIG. 4. Volume fraction $f(\mathrm{a})$, and the calculated temperature dependencies of resistivity (b) at 0 and $4 \mathrm{~T}$ magnetic fields with parameters as: $\rho_{0}=9$ $\mathrm{m} \Omega \mathrm{cm}, A=5.1 \times 10^{-7} \Omega \mathrm{cm} \mathrm{K}^{-2}, B=4.0 \times 10^{-13} \Omega \mathrm{cm} \mathrm{K}^{-5}, C=2.46$ $\times 10^{-6} \Omega \mathrm{cm} \mathrm{K}^{-1}, E_{g} / k_{B}=1500 \mathrm{~K}, T_{C}^{\bmod }=213.3 \mathrm{~K}, U_{0} / k_{B}=3750 \mathrm{~K}$ for $H=0 \mathrm{~T}$ and $\rho_{0}=9 \mathrm{~m} \Omega \mathrm{cm}, A=4.65 \times 10^{-7} \Omega \mathrm{cm} \mathrm{K}^{-2}, B=1.5 \times 10^{-13}$ $\Omega \mathrm{cm} \mathrm{K}^{-5}, C=2.46 \times 10^{-6} \Omega \mathrm{cm} \mathrm{K}^{-1}, E_{g} / k_{B}=1190 \mathrm{~K}, T_{C}^{\mathrm{mod}}=250 \mathrm{~K}$, $U_{0} / k_{B}=3750 \mathrm{~K}$ for $H=4 \mathrm{~T}$. Note that these figures refer to the $\mathrm{La}_{0.67} \mathrm{Ca}_{0.33} \mathrm{Mn}_{0.85} \mathrm{Cu}_{0.15} \mathrm{O}_{3}$ epitaxial thin film.

field increases. This can be understood as follows. Taking LCMCO film into consideration, when the temperature is above the zero field transition temperature $T_{C}^{\bmod }(0)$, the external magnetic field makes the PI regions change into the FM regions more easily, and suppresses the formation of polarons and spin-disorder scattering, leading to the monotonously decrease of $E_{g}$ and the increase of $T_{C}^{\mathrm{mod}}$, as shown in Fig. 5. The monotonous decrease of $E_{g}$ is due to the fact that the ability of the polarons to trap electrons is weakened as the spins in the polarons attempt to align along the magnetic field, which will suppress spin-disorder scattering. Since the magnetic field energy is linearly proportional to $H^{2}$, it is natural to estimate that the activation energy of the polarons decreases with $H^{2}$ for the LCMCO thin film. Indeed, the field-dependent $E_{g}$ can be fitted by

$$
E_{g}(H) / k_{B}=E_{g}(0) / k_{B}-\alpha H^{2},
$$

where $E_{g}(0)$ and $\alpha$ are fitting parameters (see the caption of Fig. 5). From Fig. 5 one may also notice that the fielddependent $T_{C}^{\text {mod }}$ for the LCMCO thin film is almost proportional to $H$ in the field range investigated, i.e.,

$$
T_{C}^{\bmod }(H)=T_{C}^{e x}(0)+\beta H .
$$

Here, $T_{C}^{e x}(0)(=226.8 \mathrm{~K})$ is the extrapolated temperature at zero field from the linear fitting, $\beta(=6.05 \mathrm{~K} / \mathrm{T})$ is a coefficient denoting $6.05 \mathrm{~K}$ per Tesla increase of $T_{C}^{\text {mod }}$ induced by 


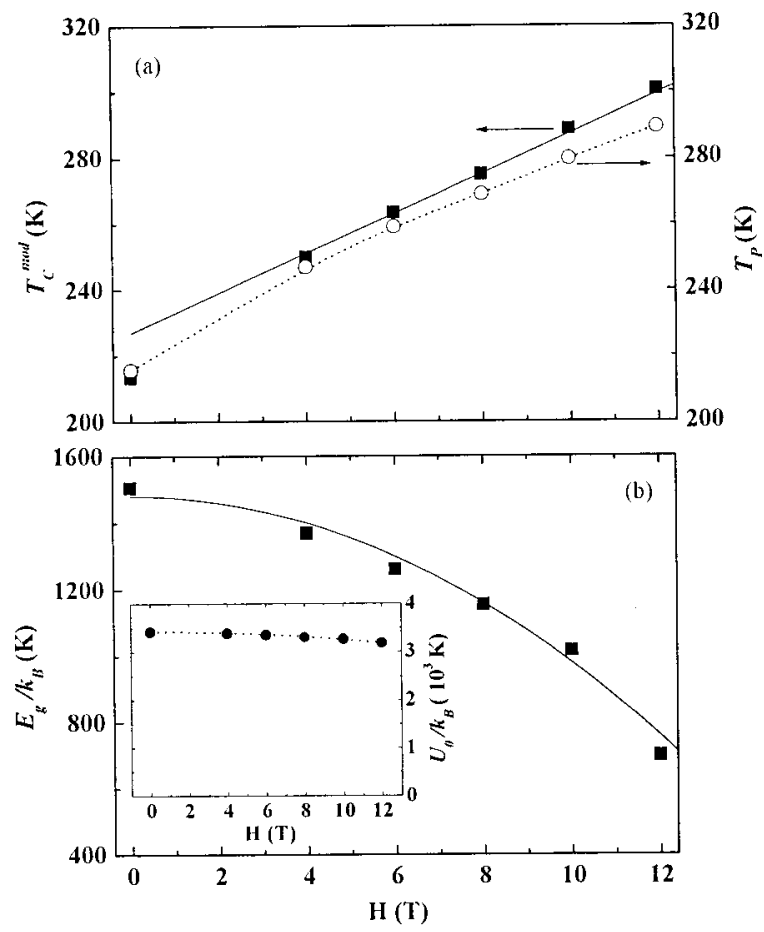

FIG. 5. Magnetic field dependencies of $T_{C}^{\mathrm{mod}}$ and $T_{P}$ (a), and $E_{g} / k_{B}$ and $U_{0} / k_{B}$ (b) which are determined from the experimental data and fitting process for the $\mathrm{La}_{0.67} \mathrm{Ca}_{0.33} \mathrm{Mn}_{0.85} \mathrm{Cu}_{0.15} \mathrm{O}_{3}$ epitaxial thin film. The solid lines are plotted according to Eqs. (4) and (5) with parameters as: $E_{g}(0) / k_{B}$ $=1480 \mathrm{~K}, \alpha=5.0 \mathrm{~K} / \mathrm{T}^{2} ; T_{C}^{e x}(0)=226.8 \mathrm{~K}$, and $\beta=6.05 \mathrm{~K} / \mathrm{T}$. The dotted lines are guide to eyes.

magnetic fields. In the low-temperature region, the applied magnetic field enhances the magnetism of the FM region and the single-magnon scattering interaction on the conducting electron is weakened, which makes $A$ become smaller.

Moreo et al. ${ }^{20}$ found that there is a robust pseudogap featuring low and intermediate temperatures, particularly at or near regimes where phase separation occurs as long as manganites have a mixed-phase characteristic. Their theoretical calculation showed that the pseudogap about $0.2-0.4 \mathrm{eV}$ is very similar to that found in a bilayered compound $\mathrm{La}_{1.2} \mathrm{Sr}_{1.8} \mathrm{Mn}_{2} \mathrm{O}_{7}$ system via high-energy resolution angleresolved photoemission measurements. In $U_{0}$ we also obtained about $0.3-0.4 \mathrm{eV}$ (see Table I), which may be viewed in some sense as an energy gap of the quasiparticles in the phase-separated FM and PI states. In our case, $U_{0}$ weakly depends on the magnetic field, as verified by the inset of Fig. 5(b) ( $U_{0}$ shows only $4 \%$ change for $H$ from 0 to $12 \mathrm{~T}$ for the LCMCO film).

Finally, we indicate that our model has been found to the applicable to those systems which exhibit a manifest PI-FM transition, but it would be invalid when some other phase transitions like antiferromagnetic insulator PI are mainly involved in the same region.

\section{CONCLUSION}

The temperature and magnetic field dependencies of resistivity were measured for $\mathrm{La}_{0.67} \mathrm{Ca}_{0.33} \mathrm{Mn}_{1-x} \mathrm{Cu}_{x} \mathrm{O}_{3}(x=0$ and 0.15 ) epitaxial films. It is observed that the competition between the FM and PI phases plays an important role in the CMR effect. Based upon a scenario that the system consists of the phase-separated FM and PI regions, a simple phenomenological model was developed. The model is well able to fit our experimental data as well as others.

\section{ACKNOWLEDGMENTS}

This work was supported by the Chinese Natural Science Foundation and the Ministry of Science and Technology of China, and in part by a CRCG Grant at the University of Hong Kong.

${ }^{1}$ R. Von Helmolt et al., Phys. Rev. Lett. 71, 2331 (1993); S. Jin et al., Science 264, 413 (1994).

${ }^{2}$ C. Zener, Phys. Rev. 81, 440 (1951); P.-G. DeGennes, ibid. 118, 141 (1960).

${ }^{3}$ A. J. Millis, P. B. Littlewood, and B. I. Shraiman, Phys. Rev. Lett. 74, 5144 (1995).

${ }^{4}$ K. H. Kim, M. Uehara, C. Hess, P. A. Sharma, and S.-W. Cheong, Phys. Rev. Lett. 84, 2961 (2000).

${ }^{5}$ N. A. Babushkina, L. M. Belova, D. I. Khomskii, K. I. Kugel, O. Yu. Gorbenko, and A. R. Kaul, Phys. Rev. B 59, 6994 (1999).

${ }^{6}$ S. Yunoki, J. Hu, A. L. Malvezzi, A. Moreo, N. Furukawa, and E. Dagotto, Phys. Rev. Lett. 80, 845 (1998); S. Q. Shen and Z. D. Wang, Phys. Rev. B 58, R8877 (1998).

${ }^{7}$ P. B. Littlewood, Nature (London) 399, 29 (1999).

${ }^{8}$ V. Kiryukhin, B. K. Kim, V. Podzorov, S.-W. Cheong, T. Y. Koo, J. P. Hill, I. Moon, and Y. H. Jeong, Phys. Rev. B 63, 24420 (2000).

${ }^{9}$ M. Jaime, P. Lin, S. H. Chun, M. B. Salamon, P. Dorsey, and M. Rubinstein, Phys. Rev. B 60, 1028 (1999).

${ }^{10}$ M. Rubinstein, J. Appl. Phys. 87, 5019 (2000).

${ }^{11}$ S. L. Yuan, J. Q. Li, G. Li, Y. Jiang, X. Y. Zeng, Y. P. Yang, and S. Z. Jin, J. Phys.: Condens. Matter 12, L337 (1999).

${ }^{12}$ S. B. Oseroff, M. Torikachvili, J. Singley, S. Ali, S. W. Cheong, and S. Schulz, Phys. Rev. B 53, 6521 (1996).

${ }^{13}$ M. Uehara, S. Mori, C. H. Chen, and S.-W. Cheong, Nature (London) 399, 560 (1999).

${ }^{14}$ J. B. Goodenough and J.-S. Zhou, Nature (London) 386, 229 (1997).

${ }^{15}$ Here we use a scenario that the percolative PI to FM transition occurs at $f=1 / 2$. This scenario is rigorous for two-dimensional systems and seems to be a good approximation even for three-dimensional systems, because $f=1 / 3$ (percolative insulator-metal transition for 3D) merely corresponds the temperature slightly above the PI-FM transition temperature.

${ }^{16}$ M. Jaime, P. Lin, M. B. Salamon, and P. D. Han, Phys. Rev. B 58, R5901 (1998).

${ }^{17}$ G.-M. Zhao, V. Smolyaniova, W. Prellier, and H. Keller, Phys. Rev. Lett. 84, 6086 (2000).

${ }^{18}$ Y. X. Jia, L. Lu, K. Khazeni, V. H. Crespi, A. Zettl, and M. I. Cohen, Phys. Rev. B 52, 9147 (1995); T. Holstein, Ann. Phys. (N.Y.) 8, 325 (1959); 8, 343 (1959); D. Emin and T. Holstein, Phys. Rev. Lett. 36, 323 (1976).

${ }^{19}$ G. Varelogiannis, Phys. Rev. Lett. 85, 4172 (2000).

${ }^{20}$ A. Moreo, S. Yunoki, and E. Dagotto, Phys. Rev. Lett. 83, 2773 (1999). 